\title{
Impression Effect vs. Click-through Effect: Mechanism Design of Online Advertising
}

\author{
Xiang Zou \\ Southeast University, China \\ zxiangr@yeah.net
}

\author{
Ruhai Wu \\ McMaster University, Canada \\ wuruhai@mcmaster.ca
}

\author{
Weijun Zhong \\ Southeast University, China \\ zhongweijun@seu.edu.cn
}

\begin{abstract}
Search advertising and display advertising are two major online advertising formats. Search advertising emphasizes ads' click-through effect. Advertisers only pay when users click the link of their ads. Traditional display advertising emphasizes ads' impression effect. Most display ads are charged based on the number of views on the ads. Considering that most online ads increase brand awareness (impression effect) and directly promote sales (click-through effect), the notemphasized effect in search advertising or display advertising actually has a significant impact on the market outcome. However, these impacts have been largely ignored. In this paper, we examine various mechanisms in search and display advertising by considering both ads' impression effect and clickthrough effect. Interestingly, we show a seesaw relationship between ads' two effects in search advertising. The advertiser whose advertisement has a strong click-through effect benefits relatively less from its impression effect. In display advertising, the realtime-bidding (RTB) mechanism considers both ads' impression effect and click-through effect. It allows a publisher to gain more surplus than that through a static auction. However, we show that RTB is associated with a high risk of market failure.
\end{abstract}

\section{Introduction}

Online advertising has grown fast to become the leading advertising format. It's estimated that internet advertising will overtake TV advertising revenue in 2017 [1]. Search advertising and display advertising are two dominant online advertising formats. Search ads, done by the search engine, are usually placed to elicit an immediate performance, especially for a click. Advertisers only pay when users click the links of their advertisements. So advertisers in search advertising are always focused on the "click-through effect" of ads. Most display ads, which are posted for publicizing a product or an upcoming event, are used to create impressions. They are always charged based on the number of views of the ads, so in display advertising; ads' impression effect is emphasized. Considering that online ads do not only increase brand awareness (impression effect) but may also directly promote sales (click-through effect), the not-emphasized effect in search advertising or display advertising, which actually has a significant impact on the market outcome, is largely ignored or covered by the shadow of the other effects. In addition, under the trend of customized advertising, a new technology, Real Time Bidding (RTB), has been introduced into traditional display advertising. That means, based on the big data of online users' information, more targeted ads are delivered via advertisers' RTB auction. RTB display advertising, represented by DoubleClick, RightMedia, has been growing so fast that the advertising spending of American is expected to reach 9.03 billion dollars by 2017, accounting for $29 \%$ of total display advertising spending [2]. Compared with traditional display advertising, it evolves from "slot buying” into "impression buying”. This new model achieves brand awareness as well as considers the ads' customization, and thus it combines two effects together and could be regarded as the third advertising format combining the traditional display advertising and search advertising.

What should be noticed is the different mechanisms of the three advertising formats. The fixed-price contract pricing scheme of traditional display advertising has changed to the individualbased real-time auction in RTB display advertising. This is also different from the static auction of search advertising. Stimulated by the two effects and from the perspective of different mechanisms in three advertising formats, we expect to explore the impact of ignored effect and examine if the newly introduced technology is more advantageous than the former ones.

In this paper, we study the cases where two advertisers compete for one advertising slot provided by a search engine (search advertising) or a publisher (display advertising). Click-through rate is a measure 
of the effectiveness of promoting sales, which is labeled as the click-through effect. The more an ad matches a user's preference, the higher chance the user has to click the link of the ad. Impression is often used as a measure of increased brand awareness. Every time an ad is displayed on a page, an impression has occurred, even if the user never clicks on it. So we assume there exists a constant impression effect each time the ad appears on a page. In search advertising, the rule of second weighted unit price is adopted to select the winners. In RTB advertising, the rule of second price auction is used. In traditional display advertising, the first price auction is adopted.

Our research shows that the ignored effect will incentivize advertisers to bid higher than when just considering the main effect and will make all players generate extra profit, especially for the platform. Therefore, the platform has an incentive to broadcast the ignored effect. Interestingly, two effects work in a consistent direction with each other in display advertising but keep a seesaw relationship in search advertising. To answer the question of mechanism, we find that the newly introduced RTB display advertising is technically and economically advanced for the platform, however, it still has the risk of system collapse.

To the best of our knowledge, this research is one of the first studies on the mechanisms of different advertising formats based on two main effects of online advertising. This work makes a contribution to the understanding of online advertising by providing a more comprehensive analysis of the feature and generality of different formats and how they affect all the players. It also makes a theoretical contribution to the emerging RTB technology by exploring its auction mechanism and bidding strategies.

The rest of the paper is organized as follows. Related literature is discussed in the next section. Then we set our basic model for the whole paper. The detailed analysis of search advertising and display advertising is in the subsequent sections. After that, an extension equilibrium analysis is offered. Finally, we get our conclusions.

\section{Literature}

The studies on online advertising and related works that we discuss in this paper, draw on three streams of literature, the relationship between search advertising and display advertising, the different auction mechanisms used in online advertising, and the new business model of RTB advertising.

On one hand, different advertising effectiveness is emphasized in this paper. Bidders in search advertising are always "performance advertisers" who place ads in order to elicit an immediate performance, whereas many display ads are placed by "brand advertisers," who are instead building awareness [3, 4]. However, advertisers could achieve two effects simultaneously no matter which kind of advertising format is adopted. Google and Ipsos MediaCT ran over 60 search experiments in 2013 in hopes of understanding how search ads affect brand awareness. As part of the studies, 800 U.S. consumers participated in the simulated-search scenarios. The study found that search ads do in fact have a positive impact on brand awareness which was published in 2014. At the same time, Fulgoni and Mörn presented that online display advertisements affected consumer behavior and that there were latency effects between branding effects and sales lifts even when click rates were minimal [5]. Only a limited number of studies examine the interaction between the two main effects and thus inspired by the practical problem and theoretical research, two effects are considered simultaneously in this paper.

Our research is also relevant to the literature on online advertising auctions. The mostly studied auction in online advertising is the one that adopted by Google. In 2002, Google started AdWords using Generalized Second Price Auction (GSP) [6, 7] and then added the ranking factor into its bidding. The factor extended from CTRs at start-up to a more comprehensive "quality score" by now [8]. In this paper, the quality score is represented by CTRs for simplification. Following Chen \& Stallaert, our research uses second weighted unit-price auctions to sell slots and assumes auctions are under a completeinformation setting [9]. Unlike most studies which focus on providing a better design of auctions in online advertising, in this paper we make it as a given tool to better understand the bidding strategy and its implication to all players.

RTB, an emerging and promising practical marketing technology, is attractive to researchers recently. In limited papers relevant to RTB and from the perspective of structure and players, the current research topics could mainly be divided into three parts [10]. Publishers and SSPs, as the supply side, constitute the first perspective. In practice, publishers usually sell premium ad inventory to high-quality advertisers via contract negotiation on an abundance of ad impressions to avoid default, the remnant ad inventory is sold through RTB to get the best matches. Hence how to allocate the different channels, how to optimize reserved price, and how to make revenue optimization are hot research questions. For instance, Balseiro et al., considers the tradeoff between the realtime revenue with the long-time benefits of traditional 
reservation-based ad contracts, formalized the combined allocation problem as multi-objective stochastic control problem and designed an efficient policy for online ad allocation [11]. Fridgeirsdottir et al. determined the publisher's optimal pricing in display advertising faced with uncertain setting [12]. Contract to supply side, the second perspective is advertisers and DSPs which are the demand side of RTB. They are willing to bid and pay for the bestmatched ad impressions using the real-time auction. Therefore, the bidding behavior and strategies are appealing to researchers and attract intensive eyeball. Balseiro et al. introduced the notion of a fluid meanfield equilibrium (FMFE) that is behaviorally appealing and computationally tractable, and in some important cases, it has a closed-form characterization. The rational behavior of advertisers could be approximated well in the context of budgetconstrained by FMFE [13]. Actually, DSPs are the proxy of advertisers which play the intermediary role between AD Exchange (AdX) and advertisers in RTB market. Each DSP chooses the highest bids from advertisers at first and then AdX picks out the highest one from different DSPs, so there exists a two-stage second price auction, and thus the selection of the appropriate algorithms and the balance of revenue between advertisers and DSPs are important issues to DSPs. As mentioned before, AdX, as the most critical component in RTB, is the marketplace where publishers sell their ads inventory and advertisers buy impressions via the auction mechanism. In this part, the key research issue is mainly about auction mechanism design. It has been proved that the optimal mechanism is second price sealed bid auction. However, there exists the problem of imperfect truthful bids submitting by DSPs because of the twostage auction. In order to deal with this problem, a mechanism called "optional second-price" (OSP) auction is introduced which practically used by Google DoubleClick[14]. Another mechanism named "BIN-TAC" is also theoretically proved to be effective $[15,16]$. These papers are mostly related to the bidding strategy of advertisers and the auction mechanism of $\mathrm{AdX}$. In order to focus on the research question, the role of DSPs is ignored and thus the auction is simplified to one-stage auction. Our research contributes to this stream of literature by comparing the different mechanisms adopted by different advertising formats and how the promising newly technology works.

\section{Model Setup}

Considering two advertisers compete for one advertising slot offered by a platform. Ads from two advertisers match each user's requirement to different degrees, at the same time the users have different preference to the advertisements which will result in different clicking probabilities on ads.

A standard Hotelling model is suggested to measure the fitness between advertisers and users. Two advertisers, indexed by $i=A, B$, stand at the opposite ends of the unit length $[0,1]$. Users, normalized to one unit, uniformly distributed along the line. The distance between a user and an advertiser describes the degree of matching between them which will be translated into different clicking probabilities. The most targeted user for an advertiser is the one whose location is the same with the advertiser. The remaining users match the advertiser decreasing with the distance. Therefore, assume $q_{i}$ is the maximum probability that the most targeted user clicks the ads from advertiser $i$. For the other users, the click probability decays along the distance with decay factor $r$. So a user located at $x$ from advertiser $A$ has the expected click-through rates of $q_{A}(1-r x)$ and $q_{B}(1-$ $r(1-x))$ from advertiser $A, B$ respectively.

Denote the unit value that advertiser $i$ derives from each click is $v_{i}$. Then Advertiser $A(B)$ has the expected value of $v_{A} p_{A}(1-r x)\left(v_{B} q_{B}(1-r(1-x))\right)$ from the user at $x$. These benefits coming from clicks on the advertisement can be regarded as the "clickthrough effect". $v_{i} q_{i}$ is their maximum expected value from the most targeted user's click. Any other user has a discount value to advertiser $i$ by the distance, which means the click-through effect decays with the degree of matching. Opposite to that, the impression effect has no relationship with the ads' fitness. Each time an ad is shown to a user, an impression occurs no matter the user clicks it or not. Suppose the impression effect is constant, the advertiser derives a value of $s$ from each view.

Two advertisers have full information about each other's value, maximum clicking probabilities $\left(q_{i}\right)$ and also have good knowledge of each users' preference which means two advertisers know the location information of each other. In addition to that, the decay factor $(r)$ is common knowledge. Two advertisers can optimize their utilities and take the best response. We will use the same basic model setup to study three advertising formats which have the different context, pricing scheme, and auction mechanisms.

\section{Search advertising}

In search advertising, suppose there is one advertising slot offered by the search engine. Each advertiser bids for the chosen keywords of ads. The search engine matches users' queries with ads' 
keywords and then assigns the ad slot to the advertiser. The mechanism used by the search engine to sell advertising slots is a second weighted unit-price auction, which is the most widely adopted approach based on Google's algorithm. Advertisers bid on cost per click (CPC) and the winner is chosen by their expected ad payments based on the cost-per-click bids. A quality score will be given to each potential ad to measure the expected number of clicks. As clickthrough rate (CTR) is the most important factor defining quality score and is highly correlated to the latter, we use CTR to represent quality score for simplification in this model. The expected ad payment is the product of the bidded CPC and the quality score / CTR. The advertiser with the largest expected payment wins the auction and pays the second largest expected payment. In the case of two advertisers' auction, the one who wins the auction will pay the other one's proposed payment.

According to the pricing scheme and payment rule, advertisers pay only for clicks, not impressions. So in search advertising, the click-through effect is the main effect. Actually, the impression effect also plays a role because of the display behavior on the search result webpages no matter the user clicks it or not. However, it hasn't been charged by the search engine and is often ignored in the previous papers. If the impression effect is taken into account, the extra effect will influence advertisers' bidding strategies and then result in profit changes for the search engine and two advertisers.

As in the basic model, suppose the impression effect is $s$ and two advertisers preset the bid price $b_{i}$, each time the user $x$ clicks on the ads from two advertisers, the advertisers are expected to pay $b_{A} q_{A}(1-r x)$ or $b_{B} q_{B}(1-r(1-x))$ to the search engine respectively. The marginal user $\bar{x}$ who is no difference to the search engine when bidding $b_{A}, b_{B}$ is:

$$
\begin{array}{r}
b_{A} q_{A}(1-r \bar{x})=b_{B} q_{B}(1-r(1-\bar{x})) \\
\bar{x}=\frac{b_{A} q_{A}-b_{B} q_{B}(1-r)}{\left(b_{A} q_{A}+b_{B} q_{B}\right) r}
\end{array}
$$

With two advertisers' bidding price, the search engine segments the whole market into two parts. The users in the interval $[0, \bar{x}]$ will be targeted to advertiser $A$ 's advertisement, which is $A$ 's market share; on the other hand, $[\bar{x}, 1]$ is the market share for advertiser $B$.

For advertisers $A, B$, their expected profits come from three parts, the first one represents click-through effect, the second is impression effect and the last one is the expected payment of advertisers.

$$
\begin{aligned}
\pi_{A}= & \int_{0}^{\bar{x}} v_{A} q_{A}(1-r x) d x+\int_{0}^{\bar{x}} s d x \\
& -\int_{0}^{\bar{x}} b_{B} q_{B}(1-r(1-x)) d x \\
\pi_{B}= & \int_{\bar{x}}^{1} v_{B} q_{B}(1-r(1-x)) d x+\int_{\bar{x}}^{1} s d x
\end{aligned}
$$

$$
-\int_{\bar{x}}^{1} b_{A} q_{A}(1-r x) d x
$$

Lemma 1: Equilibrium bidding strategies are

$$
\begin{aligned}
& b_{A}=v_{A}\left(1+\frac{s}{2-r}\left(\frac{1}{v_{B} q_{B}}+\frac{1}{v_{A} q_{A}}\right)\right) \\
& b_{B}=v_{B}\left(1+\frac{s}{2-r}\left(\frac{1}{v_{B} q_{B}}+\frac{1}{v_{A} q_{A}}\right)\right)
\end{aligned}
$$

And the larger the impression effect, the larger the bid which means the ignored impression effect will push advertisers to bid higher to win the auction.

Lemma 1 also shows Advertisers' optimal bids are proportional to their values.

1) $s=0, b_{i}=v_{i}$. If two advertisers do not recognize the benefit of impression effect, or they haven't put the impression effect into consideration in their bidding strategies, the advertisers bid their true values. This result is consistent with the claims of previous literature and can be regarded as the benchmark case.

2) $s>0, b_{i}>v_{i}$. When impression effect is considered by two advertisers, the impression effect pushes advertisers to bid higher than their values to win the auction. The increased bidding values are proportional to the benefit of impression effect and affected by the click-through effects of both advertisers as well.

Lemma 2: Impression effect will not affect the market share/coverage of the two ads when impression effect is constant to all visitors.

The location of the marginal user has not changed with and without of the impression effect:

$$
\bar{x}=\frac{b_{A} q_{A}-b_{B} q_{B}(1-r)}{\left(b_{A} q_{A}+b_{B} q_{B}\right) r}=\frac{v_{A} q_{A}-v_{B} q_{B}(1-r)}{\left(v_{A} q_{A}+v_{B} q_{B}\right) r}=\bar{x}^{\prime}
$$

If considering only the click-through effect, each user has a different probability of making a contribution $v_{A}, v_{B}$ to the search engine respectively. For users in the left part $\left[0, \bar{x}^{\prime}\right]$, they generate more revenue when showing ads from advertiser $A$ rather than $B$; vice versa. In that case, the market shares are determined by the search engine based on the marginal user. No changes in market share when considering the extra impression effect is very intuitive in that the utility of impression effect is the same for two advertisers so they have an equivalent increase in the bidding price in equilibrium. And the market share is positively related to its own click-through, but negatively correlated to its competitors'.

Lemma 3: There is a seesaw relationship between impression effect and click-through effect in search advertising.

Denote $1+\frac{s}{2-r}\left(\frac{1}{v_{B} q_{B}}+\frac{1}{v_{A} q_{A}}\right)$ as $M$. An advertiser's optimal bid is $M$ times of the value that the advertiser gains from each click. The increase of bidding price results from the extra impression effect. From this sense, $M$ can be regarded as the multiplier 
of impression effect. From the mathematical expression, on one hand, $M$ increases with $s$; on the other hand, $M$ has a negative correlation with, $v_{A} q_{A}$ and $v_{B} q_{B}$, in which $v_{i} q_{i}$ can be seen as the indicator of click-through effect for the advertisers. Therefore, there is a seesaw relationship between impression effect and click-through effect. If the click-through effect is more important to the advertiser, the impression effect will lower down and correspondingly the increased degree of bids for the slot is not that much.

Table 1 (see Appendix) summarizes the profits of two advertisers and the search engine when considering the impression effect or not.

Proposition 1: When considering the impression effect in search advertising, the platform and two advertisers share the increased profit. The platform increases the higher profit percentage than two advertisers and the advertiser whose advertisement has a strong click-through effect benefits relatively less from the advertisement's impression effect.

From the analysis above, we can find that the original profits of two advertisers without impression effect are increasing with their own click-through effect and decreasing with the opponent's. This means that when click-through effect is the only consideration, the two advertisers compete on the click-through effect and the one with the higher clickthrough effect gains a larger size market segment. When impression effect is considered, the profit of the platform (search engine) and two advertisers are all increased, they share the increased surplus resulting from the impression effect $\left(\Delta \pi_{A S}, \Delta \pi_{B S}, \Delta \pi_{P S}>0\right)$.

The increased percentage of profit $\frac{\Delta \pi_{A S}}{\pi_{A S}^{\prime}}, \frac{\Delta \pi_{B S}}{\pi_{B S}^{\prime}}, \frac{\Delta \pi_{P S}}{\pi_{P S}^{\prime}}$ which is positive to the unit value $s$ can also be viewed as the result of the impression effect, among them the platform increases the highest profit percentage (which is the sum of two advertisers $\frac{\Delta \pi_{P S}}{\pi_{P S}^{\prime}}=\frac{\Delta \pi_{A S}}{\pi_{A S}^{\prime}}+$ $\left.\frac{\Delta \pi_{B S}}{\pi_{B S}^{\prime}}\right)$ ) than two advertisers. Numerically, the platform also increases up to $M$ times of $\pi_{P S}{ }^{\prime}$. The benefit which results from higher bidding price is all directly transferred into the platform, so it's understandable that the platform increases the same degree $M$ with the bidding price. As for two advertisers, on one hand, they benefit from the impression effect, on the other hand, the payment also rises because of the opponent's higher bids, so the increased percentages of two advertisers are lower than the platform. Another interesting finding is that the increased percentage is an inverse correlation to the click effect whereby the platform is negatively related to both of advertisers' click effects and each advertiser has a negative relationship with its own click effect respectively.
That means there is a larger increased percentage for an advertiser who has a lower click effect, so the small advertiser will benefit more when considering the impression effect. It's proven that there is a tradeoff between click effect and impression effect.

From the perspective of the platform, it has been proven theoretically that the platform has incentives to broadcast the impression effect accompanied with the search advertising results, it will increase profit for all the participants, especially for itself.

\section{Display advertising}

Display advertising is another important online advertising format. This kind of advertising is done by the web publishers who post the advertisement on their websites. With technology improving, display advertising has evolved from the traditional display into real-time bidding display advertising. Although the main purpose of display advertising is delivering general advertisements or events to create or maintain brand awareness, these two types have very different mechanisms to show the ads.

\subsection{Traditional display advertising}

In traditional display advertising, advertisers would purchase ad slots in bulk in a certain period of time by making contracts directly with publishers. The same ads with no target will be shown to all visitors. The advertisers are charged based on the number of views of the ads, therefore impression effect is the main consideration for advertisers and publishers. Even though it's used mainly for awareness and appearance, it has the probability to attract users' interests, clicks, and even future buying. Unfortunately, click-through effect is not frequently considered in display advertising.

Suppose two advertisers compete for an appealing ad slot on a website. The basic model setup still works for display advertising. What should have been noticed is the payment. It's the first price auction instead of second price auction in traditional display advertising which means the publisher chooses the advertiser with the highest bid to sell the ad slot.

Lemma 4 When only considering impression effect $s$, two advertisers are both willing to pay $s$ which is the true value for the slot and get zero profit. So there is no difference for the platform to assign the slot to any advertiser and the profit of the platform is $S$.

$$
\begin{gathered}
b_{A T}^{\prime}=b_{B T}^{\prime}=s \\
\pi_{A T}^{\prime}=\pi_{B T}^{\prime}=0, \pi_{P T}^{\prime}=s
\end{gathered}
$$


When considering the benefit of click-through effect in traditional display advertising, the new values of two advertisers are respectively:

$$
\begin{aligned}
& V_{A}=\int_{0}^{1} s d x+\int_{0}^{1} v_{A} q_{A}(1-r x) d x \\
& =s+v_{A} q_{A}\left(1-\frac{1}{2} r\right) \\
& V_{B}=\int_{0}^{1} s d x+\int_{0}^{1} v_{B} q_{B}(1-r(1-x)) d x \\
& =s+v_{B} q_{B}\left(1-\frac{1}{2} r\right)
\end{aligned}
$$

According to the rule of first price auction in traditional advertising, if $v_{A} p_{A}>v_{B} q_{B}$, advertiser $B$ will not bid more than $s+v_{B} q_{B}\left(1-\frac{1}{2} r\right)$, so that advertiser A only needs to bid a little more than $B$ to win the auctions $+v_{B} q_{B}\left(1-\frac{1}{2} r\right)+\varepsilon$; and if $v_{B} q_{B}>$ $v_{A} p_{A}, B$ will bid $\mathrm{s}+v_{A} q_{A}\left(1-\frac{1}{2} r\right)+\varepsilon$ to be the winner. The advertiser with higher click-through effect will post a higher bid than $s$ and acquire extra profit. The platform is expected to acquire more profit $\mathrm{s}+v_{i} q_{i}\left(1-\frac{1}{2} r\right)+\varepsilon$ instead of $s$. At this time, two effects work consistently with each other. This is in contrast to the relationship in the search advertising.

$$
\begin{aligned}
& \text { If } v_{A} q_{A}>v_{B} q_{B} \\
& \pi_{A T}=\left(v_{A} q_{A}-v_{B} q_{B}\right)\left(1-\frac{1}{2} r\right)-\varepsilon, \pi_{B T}=0 \\
& \pi_{P T}=s+v_{B} q_{B}\left(1-\frac{1}{2} r\right)+\varepsilon \\
& \text { If } v_{B} q_{B}>v_{A} q_{A} \\
& \pi_{A T}=0, \pi_{B T}=\left(v_{B} q_{B}-v_{A} q_{A}\right)\left(1-\frac{1}{2} r\right)-\varepsilon \\
& \pi_{P T}=s+v_{A} q_{A}\left(1-\frac{1}{2} r\right)+\varepsilon
\end{aligned}
$$

Lemma 5 When considering click-through effect in traditional display advertising, the winning bid will be higher. Hence, the platform is expected to acquire more profit than $s$.

\subsection{RTB display advertising}

Under RTB display advertising, the ad slot would be sold in real-time to the highest bidder based on each individual impression via the auction mechanism. The "slot buying" of traditional display advertising has changed into "individual impression buying" of RTB advertising, therefore users with different preferences will be shown different ads. Although they both use an auction mechanism to determine the winner, there is a difference between search advertising's keyword auction and individual-oriented auction in RTB display advertising. In the keyword auction, it is a onebidding action, while in RTB, the advertisers are competing for each visitors' impression. The second difference among them is that it's the advertisers themselves who choose to take part in each auction with RTB display advertising, but for other two formats, the allocation is made by the platform.

RTB, which matches ads with users on an individual basis, brings the chance to consider not only the impression effect of the traditional display, but also naturally takes users' click-through action into consideration. In that way, the bidding strategies of advertisers will change accordingly.

Suppose two advertisers bid $b_{A x}, b_{B x}$ for each display opportunity. Click-through effects and impression effects are denoted as the same as in the model of search advertising. Following the literature [9], when considering two effects simultaneously in RTB advertising, we consider the weakly dominant bidding strategies by the advertisers.

Lemma 6: In the interval of $[0, \hat{x}]$, advertiser $A$ always bids higher than advertiser $B, b_{A x}>b_{B x}$, bidding its total true value is advertiser $B$ 's weakly dominant strategy which is $s+v_{B} q_{B}(1-r(1-x))$; opposite to that, in the interval $[\hat{x}, 1]$, advertiser $B$ bids higher than advertiser $A, b_{B x}>b_{A x}$, and advertiser $A$ 's weakly dominant strategy is bidding its true value $s+v_{A} q_{A}(1-r x)$.

When advertisers auction off each impression to show ads to individuals, the bids stem from two parts: the part of $\mathrm{s}$ is completely transferred from impression effect into the bid, which means the impression effect doesn't influence the bids in RTB display advertising. The other part coming from the click-through effect, is the expected value of one click. Or it could be viewed as the total value $v_{i} q_{i}$ discounted by the distance, so the more precise the user matching the advertiser, the higher the advertiser's bid to the user.

Lemma 7: When considering two effects in RTB display advertising, it achieves the same market segmentation with that in search advertising, although they have different auction mechanisms.

The marginal user in RTB display advertising is $\hat{x}=\frac{v_{A} q_{A}-v_{B} q_{B}(1-r)}{\left(v_{A} q_{A}+v_{B} q_{B}\right) r}$. The marginal user is the same as that in search advertising. In search advertising, the search engine assigns the users according to the advertisers' bids multiplied by the click-through rates. In RTB, the two advertisers bid based on each user's characteristic. Although they have different paradigms, they bring out the same segmentation results.

The profits of two advertisers and the platform when considering two effects, are:

$$
\begin{aligned}
\pi_{A R}= & \int_{0}^{\bar{x}} s d x+\int_{0}^{\bar{x}} v_{A} q_{A}(1-r x) d x \\
& -\int_{0}^{\bar{x}} b_{B x} d x \\
\pi_{B R}= & \int_{\bar{x}}^{1} s d x+\int_{\bar{x}}^{1} v_{B} q_{B}(1-r(1-x)) d x \\
& -\int_{\bar{x}}^{1} b_{A x} d x
\end{aligned}
$$




$$
\pi_{P R}=\int_{0}^{\bar{x}} b_{B x} d x+\int_{\bar{x}}^{1} b_{A x} d x
$$

Lemma 8 If impression effect is the only effect taken into account in RTB display advertising, two advertisers will have the same bids, which equal to the value of the impression $s$. Consequently their expected profits are zero from each auction. The platform's expected profit is $s$.

If only impression effect is considered in RTB display advertising, the equilibrium outcome is the same as that in the traditional display advertising.

Proposition 2 When considering two effects in RTB display advertising, the bidding price increases, the platform and two advertisers all generate extra profit compared with the case when only considering the impression effect. What's more important, the advanced advertiser with higher click-through effect benefits more than the one with lower click-through effect.

When comparing the bidding strategy and the profits without and with click-through effect under RTB (see Table 2 in Appendix), the extra effect pushes two advertisers to bid aggressively than before. The bids are not blind but correlated to users' characteristics and click-through effect. Furthermore, the increased payments which result from the increased bids by advertisers benefit the platform. The platform and two advertisers all acquire increased profit and thus achieve Pareto improvement because of the ignored click-through effect $\left(\Delta \pi_{A}, \Delta \pi_{B}, \Delta \pi_{P}>\right.$ $0)$. As a consequence, when the platform uses RTB, the ads are forced to be targeted. The targeting makes the click-through effect intensively added on the impression effect which results in higher bidding price and then more profits for the platform and advertisers. It forms a dynamic loop from ads targeting to bids and leading to profit. Another notable thing is the increased profit of each advertiser is positively related to its own click-through effect and inversely related to the opponent's, so it's more beneficial to the advanced advertiser with higher click-through effect than the advertiser with the lower one. And for the platform, it's understandable to recognize the consistent direction of two effects which is in contrast to the inverse correlation conclusion in search advertising.

\section{RTB- Combination of two advertising formats}

Corollary 1: An interesting finding is that RTB is a perfect combination of search advertising and traditional display advertising, which only considers their main effects, or it could be said RTB is a perfect combination of two effects.

From table 3 (see Appendix), it can be seen that the platform and two advertisers' profits under RTB are the sum of the profits in search advertising without impression effect and the profits in traditional display advertising without click-through effect. That means the newly introduced RTB technology considers and combines two effects perfectly. Different advertising formats have the tendency towards integration.

\section{Comparison of different advertising formats under two effects}

When considering two effects in three advertising formats, the extra effect will stimulate the advertiser to bid higher than when just considering one effect. However, from the perspective of mechanism design, search advertising and display advertising are essentially different. In search advertising, advertisers preset the bidding price for keywords and then the search engine allocates the ad placement to two advertisers which is a one-shot action. As to traditional display advertising, the publisher allocates the ad slot to only one advertiser. The principle is getting all or nothing at all. In RTB display advertising, advertisers themselves decide to auction for each user impression which is a dynamic behavior.

Lemma 9 From the angle of the platform, the profits are all increased under three cases when considering two effects, but $\pi_{P T}>\pi_{P R}>\pi_{P S}$.

First prove $\pi_{P R}>\pi_{P S}$. In search advertising, the extra profit from impression effect is shared by all players, including two advertisers and the search engine, but in RTB display advertising, the platform acquires the total profit which is completely transferred, not only from the impression effect but also from the click-through effect (Corollary 1). This means, using RTB auction, based on individuals, is a more beneficial way to improve profit compared with the classical static auction in search advertising. RTB changes the auction mechanism in online advertising, and it allows the platform to gain more surplus than that through a static auction.

Then we compare the profits in two display advertising formats and can prove $\pi_{P T}>\pi_{P R}$. Actually, it's the first price auction in traditional display advertising, but a variant of the second price auction in RTB which results in platform's lower profit. It's reasonable to understand that the premium inventory of display advertising is still using traditional advertising, and the remnant inventory is using RTB.

As for advertisers, the results are inversed under three formats $\pi_{A T}<\pi_{A R}<\pi_{A S}$. In traditional display advertising, the advertiser's profit is either o or very small, in RTB the advertisers share the part of profit coming from the click-through effect, and in search advertising they also share the part of profit from the impression effect, which results in a more total profit 
than that in RTB display advertising. As a whole, search advertising is more beneficial to advertisers than display advertising.

Proposition 3: RTB completely changes the auction mechanism in online advertising, and it allows a platform to gain more surplus than that with a static auction, but it still has some weakness compared with the traditional display advertising. From the perspective of advertisers, it's better for them to choose search advertising rather than display advertising. (see Table 4 in Appendix)

\section{Extension about the equilibrium results}

When considering two effects in RTB display advertising, the weakly dominant strategy is rational and of great significance. Two advertisers bid for each impression to show ads on the ad slot, as a result, the whole market is segmented into two parts. In the part that is more correlated to $A$ 's ads, advertiser $B$ bids its true value which is similar to the result in the private second-price auction. Advertiser $A$ only needs to bid higher than $B$ 's true value and will win the auction. So for the platform, the bid of the advertiser with smaller click-through effect is equal to the lower bound of the bids. This weakly dominant equilibrium looks perfect for all the parties including the platform and two advertisers. However, the equilibrium which we get in the former analysis is just one of equilibriums, it's not a general equilibrium. From the general equilibrium, we find that it may bring out system collapse due to the low bid of the opponent.

Lemma 10: In the interval $[0, \hat{x}]$, the equilibrium bidding strategies of two advertisers are $\left\{\left(b_{A x}, b_{B x}\right) \mid b_{A x} \in\left[s+v_{B} q_{B}(1-r(1-x)),+\infty\right)\right.$, $\left.b_{B x} \in\left[0, s+v_{A} q_{A}(1-r x)\right], b_{A x} \geq b_{B x}\right\}$.

Advertiser $A$ always bids higher or at least equal to advertiser $B\left(b_{A x}>b_{B x}\right)$, and thus advertiser $A$ wins the auction. Under this situation, advertiser $B$ has a possibility of bidding a low price (maybe zero) to give up the auction. Follow the same logic, in the interval $[\hat{x}, 1]$, advertiser $B$ bids higher or at least equal to advertiser $A$ and wins the auction, in that case, advertiser $A$ may have no incentive to take part in the auction.

According to the equilibrium strategies above, although RTB can help to increase the accuracy and efficiency of ads delivery, it may result in a situation where it decreases the advertisers' competition in an auction and even leads to system collapse. This situation is demonstrated in practical. For instance, empirical research shows that most impressions sold in Microsoft AdECN platform can only be matched to one to three advertisers. Those impressions that are matched to exactly one advertiser will be sold at their reserve prices (may be zero). This significantly reduces the revenue of AdXs and DSPs, who as a result have no incentives to segment their target audiences via big-data analysis. In literature, it has been empirically proven that the average price of impressions first rises and then drops with market segmenting. It could be found that the weakly dominant equilibrium in Part 5.2 is an ideal result for all parties, there are still possibilities of disappointing consequences. Measures, such as reserved price needs to be introduced to avoid systemic collapse.

\section{Conclusion}

In search advertising, click-through effect is emphasized for its effectiveness and tractability; but in display advertising, players usually focus on the impression effect. In this paper, we pick up the ignored effect in different advertising formats. It's found that the extra effect will push advertisers to bid higher than when just considering one effect, and the profits of all players, including the platform, increase. An interesting seesaw relationship between click-through effect and impression effect is illustrated in search advertising. The advertiser with lower click-through effect gains a smaller market segment through auction, but receives more compensation when impression effect is taken into consideration in the auction. However, in RTB display advertising, the advertiser with higher click-through effect will benefit more by considering both effects. To sum up, from the platform's perspective, it's necessary to point out the previously ignored effect which may provide an increased profit.

From the view of mechanism design, the introduction of RTB is economically advanced. The individual based real-time auction mechanism makes it a perfect combination of two effects and produces the roles of two advertising formats, so that it allows a platform to gain more surplus than that through a static auction. However, it still has some weakness and may have the risk of system collapse.

As one of the first papers to explore ignored advertising effect in online advertising, our study has several limitations that future research could address. First, we use Hotelling model to describe the bidding competition between two advertisers. It will be interesting to examine the bidding strategies by three or more advertisers, and whether the similar results remain satisfied. Second, this research has not considered the reserved price mechanism, which is prevalently adopted in online advertising auctions. Furthermore, this paper provides theoretical 
equilibrium outcomes under the three online advertising formats. It would be better to empirically examine the results with real data.

\section{Acknowledgments}

We thank the editor and the reviewers for their insightful and constructive comments. We also thank participants at the Workshop on ISMS Marketing Science Conference (2016), and seminar participants at McMster University. This study was supported by the National Natural Science Foundation of China (No. 71371050)

\section{References}

[1] Global entertainment and media outlook 2015-2019, PwC, 2015.

[2] http://www.emarketer.com/Article/RTB-Ad-SpendContinues-Robust-Growth/1009783, eMarketer, 2013.

[3] N. Arnosti, M. Beck, and P.R. Milgrom, "Adverse Selection and Auction Design for Internet Display Advertising”, Available at SSRN 2603336, 2016.

[4] M. Braun, W.W. Moe, "Online display advertising: Modeling the effects of multiple creatives and individual impression histories”, Marketing Science, 32(5), 2013, pp. 753-767.

[5] G.M. Fulgoni, M.P Mörn, "Whither the click? How online advertising works", Journal of Advertising Research, 49(2), 2009, pp. 134-142.

[6] B. Edelman, M. Ostrovsky, and M. Schwarz, "Internet advertising and the generalized second price auction: Selling billions of dollars worth of keywords", American Economic Review, 97(1), 2007, pp. 242-259.
[7], H.R. Varian, "Position auctions”, International Journal of Industrial Organization, 25(6), 2007, pp. 1163-1178.

[8] D. Liu, J. Chen, and A.B. Whinston, "Ex ante information and the design of keyword auctions", Information Systems Research, 21(1), 2010, pp. 133-153.

[9] J. Chen, J. Stallaert, “An Economic Analysis of Online Advertising Using Behavioral Targeting”, MIS Quarterly, 38(2), 2014, pp. 429-449.

[10] Y. Yuan, F. Wang, J. Li, and R. Qin, “A survey on real time bidding advertising”, IEEE International Conference on Service Operations and Logistics, and Informatics, IEEE, 2014, pp. 418-423.

[11] S.R. Balseiro, J. Feldman, V. Mirrokni, and S. Muthukrishnan, "Yield Optimization of Display Advertising with Ad Exchange”, Management Science, 60(12), 2014, pp. 2886-2907.

[12] S. Najafi-Asadolahi, K. Fridgeirsdottir "Cost-per-click pricing for display advertising”, Manufacturing \& Service Operations Management, 16(4), 2014, pp. 482-497.

[13] S.R. Balseiro, O. Besbes, and G.Y. Weintraub, "Repeated auctions with budgets in ad exchanges: Approximations and design”, Management Science, 61(4), 2015, pp. 864-88.

[14] Y. Mansour, S. Muthukrishnan, and N.Nisan, "Doubleclick ad exchange auction", ArXiv preprint arXiv: 1204.0535, 2012.

[15] L. E. Celis, G. Lewis, M. Mobius, and H. Nazerzadeh, "Buy-it-now or Take-a-chance: A New Pricing Mechanism for Online Advertising”, working paper, 2012.

[16] L. E. Celis, G. Lewis, M. Mobius, and H. Nazerzadeh, "Buy-It-Now or Take-a-Chance: Price Discrimination through Randomized Auctions”, Management Science, 60(12), 2014, pp. 2927-2948.

\section{Appendix}

Table 1. Profits without and with impression effect in search advertising

\begin{tabular}{|c|c|c|c|}
\hline & $\begin{array}{c}\text { Without impression } \\
\left(s=0, b_{i}=v_{i}\right)\end{array}$ & $\begin{array}{c}\text { With impression } \\
\left(s>0, b_{i}>v_{i}\right)\end{array}$ & Difference $(\Delta)$ \\
\hline$\pi_{A S}^{\prime}\left(\pi_{A S}\right)$ & $\frac{\left(v_{A} q_{A}-v_{B} q_{B}+v_{B} q_{B} r\right)^{2}}{2\left(v_{A} q_{A}+v_{B} q_{B}\right) r}$ & $\left(1+\frac{s}{2-r} \frac{1}{v_{A} q_{A}}\right) \pi_{A S}^{\prime}$ & $\frac{s}{(2-r)\left(v_{A} q_{A}\right)} \pi_{A S}^{\prime}$ \\
\hline$\pi_{B S}^{\prime}\left(\pi_{B S}\right)$ & $\frac{\left(v_{B} q_{B} v_{A} q_{A}+v_{A} q_{A} r\right)^{2}}{2\left(v_{A} q_{A}+v_{B} q_{B}\right) r}$ & $\left(1+\frac{s}{2-r} \frac{1}{v_{B} q_{B}}\right) \pi_{B S}^{\prime}$ & $\frac{s}{(2-r)\left(v_{B} q_{B}\right)} \pi_{B S}^{\prime}$ \\
\hline$\pi_{P S}^{\prime}\left(\pi_{P S}\right)$ & $\frac{2 v_{A} q_{A} v_{B} q_{B}\left(1-\frac{1}{2} r^{2}\right)-(1-r)^{2}\left(v_{A}{ }^{2} q_{A}{ }^{2}+v_{B}{ }^{2} q_{B}{ }^{2}\right)}{2\left(v_{A} q_{A}+v_{B} q_{B}\right) r}$ & $\left(1+\frac{s}{2-r}\left(\frac{1}{v_{A} q_{A}}+\frac{1}{v_{B} q_{B}}\right)\right) \pi_{P S}^{\prime}$ & $\frac{s}{2-r}\left(\frac{1}{v_{A} q_{A}}+\frac{1}{v_{B} q_{B}}\right) \pi_{P S}^{\prime}$ \\
\hline
\end{tabular}

Table 2. Profits without and with click-through effect in RTB display advertising

\begin{tabular}{|l|c|c|c|}
\hline & $\begin{array}{c}\text { Only } \\
\text { impression }\end{array}$ & With click-through effect & Difference $(\Delta)$ \\
\hline$\pi_{A R}^{\prime}\left(\pi_{A R}\right)$ & 0 & $\frac{\left(v_{A} q_{A}-v_{B} q_{B}(1-r)\right)^{2}}{2\left(v_{A} q_{A}+v_{B} q_{B}\right) r}$ & $\frac{\left(v_{A} q_{A}-v_{B} q_{B}(1-r)\right)^{2}}{2\left(v_{A} q_{A}+v_{B} q_{B}\right) r}$ \\
\hline$\pi_{B R}^{\prime}\left(\pi_{B R}\right)$ & 0 & $\frac{\left(v_{B} q_{B}-v_{A} q_{A}(1-r)\right)^{2}}{2\left(v_{A} q_{A}+v_{B} q_{B}\right) r}$ & $\frac{\left(v_{B} q_{B}-v_{A} q_{A}(1-r)\right)^{2}}{2\left(v_{A} q_{A}+v_{B} q_{B}\right) r}$ \\
\hline$\pi_{P R}^{\prime}\left(\pi_{P R}\right)$ & $S$ & $s+\frac{2 v_{A} q_{A} v_{B} q_{B}\left(1-\frac{1}{2} r^{2}\right)-\left(v_{A}^{2} q_{A}^{2}+v_{B}^{2} q_{B}^{2}\right)(1-r)^{2}}{2\left(v_{A} q_{A}+v_{B} q_{B}\right) r}$ & $\frac{2 v_{A} q_{A} v_{B} q_{B}\left(1-\frac{1}{2} r^{2}\right)-\left(v_{A}^{2} q_{A}^{2}+v_{B}^{2} q_{B}^{2}\right)(1-r)^{2}}{2\left(v_{A} q_{A}+v_{B} q_{B}\right) r}$ \\
\hline
\end{tabular}


Table 3. Relationship between RTB and two original advertising formats

\begin{tabular}{|c|c|c|}
\hline Search without impression & $\begin{array}{c}\text { Display without } \\
\text { click }\end{array}$ & RTB Display \\
\hline$\frac{\left(v_{A} q_{A}-v_{B} q_{B}+v_{B} q_{B} r\right)^{2}}{2\left(v_{A} q_{A}+v_{B} q_{B}\right) r}$ & 0 & $\frac{\left(v_{A} q_{A}-v_{B} q_{B}+v_{B} q_{B} r\right)^{2}}{2\left(v_{A} q_{A}+v_{B} q_{B}\right) r}$ \\
\hline$\frac{\left(v_{B} q_{B}-v_{A} q_{A}+v_{A} q_{A} r\right)^{2}}{2\left(v_{A} q_{A}+v_{B} q_{B}\right) r}$ & 0 & $\frac{\left(v_{B} q_{B} v_{A} q_{A}+v_{A} q_{A} r\right)^{2}}{2\left(v_{A} q_{A}+v_{B} q_{B}\right) r}$ \\
\hline$\frac{2 v_{A} q_{A} v_{B} q_{B}\left(1-\frac{1}{2} r^{2}\right)-(1-r)^{2}\left(v_{A}{ }^{2} q_{A}{ }^{2}+v_{B}{ }^{2} q_{B}{ }^{2}\right)}{2\left(v_{A} q_{A}+v_{B} q_{B}\right) r}$ & $S$ & $s+\frac{2 v_{A} q_{A} v_{B} q_{B}\left(1-\frac{1}{2} r^{2}\right)-\left(v_{A}^{2} q_{A}^{2}+v_{B}^{2} q_{B}^{2}\right)(1-r)^{2}}{2\left(v_{A} q_{A}+v_{B} q_{B}\right) r}$ \\
\hline
\end{tabular}

Table 4. Comparison of different advertising formats under two effects

\begin{tabular}{|c|c|c|c|c|}
\hline & & \multirow{2}{*}{ Search advertising } & \multicolumn{2}{|c|}{ Display Advertising } \\
\hline & & & Traditional & RTB advertising \\
\hline \multirow{3}{*}{ 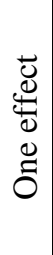 } & $\pi_{A}$ & $\frac{\left(v_{A} q_{A}-v_{B} q_{B}+v_{B} q_{B} r\right)^{2}}{2\left(v_{A} q_{A}+v_{B} q_{B}\right) r}$ & 0 & 0 \\
\hline & $\pi_{B}$ & $\frac{\left(v_{B} q_{B-} v_{A} q_{A}+v_{A} q_{A} r\right)^{2}}{2\left(v_{A} q_{A}+v_{B} q_{B}\right) r}$ & 0 & 0 \\
\hline & $\pi_{P}$ & $\frac{2 v_{A} q_{A} v_{B} q_{B}\left(1-\frac{1}{2} r^{2}\right)-(1-r)^{2}\left(v_{A}^{2} q_{A}^{2}+v_{B}^{2} q_{B}^{2}\right)}{2\left(v_{A} q_{A}+v_{B} q_{B}\right) r}$ & $S$ & $S$ \\
\hline \multirow{3}{*}{ 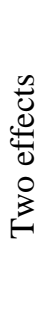 } & $\pi_{A}$ & $\left(1+\frac{s}{2-r} \frac{1}{v_{A} q_{A}}\right) \pi_{A S}^{\prime}$ & $\begin{array}{c}0 / \\
\left(v_{A} q_{A}-v_{B} q_{B}\right)\left(1-\frac{1}{2} r\right)-\varepsilon\end{array}$ & $\frac{\left(v_{A} q_{A}-v_{B} q_{B}+v_{B} q_{B} r\right)^{2}}{2\left(v_{A} q_{A}+v_{B} q_{B}\right) r}$ \\
\hline & $\pi_{B}$ & $\left(1+\frac{s}{2-r} \frac{1}{v_{B} q_{B}}\right) \pi_{B S}^{\prime}$ & $\begin{array}{c}\left(v_{B} q_{B}-v_{A} q_{A}\right)\left(1-\frac{1}{2} r\right)-\varepsilon \\
/ 0\end{array}$ & $\frac{\left(v_{B} q_{B-} v_{A} q_{A}+v_{A} q_{A} r\right)^{2}}{2\left(v_{A} q_{A}+v_{B} q_{B}\right) r}$ \\
\hline & $\pi_{P}$ & $\left(1+\frac{s}{2-r}\left(\frac{1}{v_{A} q_{A}}+\frac{1}{v_{B} q_{B}}\right)\right) \pi_{P S}^{\prime}$ & $\mathrm{s}+v_{i} q_{i}\left(1-\frac{1}{2} r\right)+\varepsilon$ & $\begin{array}{l}s \\
+\frac{2 v_{A} q_{A} v_{B} q_{B}\left(1-\frac{1}{2} r^{2}\right)-\left(v_{A}^{2} q_{A}^{2}+v_{B}^{2} q_{B}^{2}\right)(1-r)^{2}}{2\left(v_{A} q_{A}+v_{B} q_{B}\right) r}\end{array}$ \\
\hline
\end{tabular}

